\title{
¿Hay evaluación formativa y compartida en la formación inicial del profesorado en Chile? Percepción de alumnado, profesorado y egresados de una universidad
}

\author{
Francisco Javier GALLARDO-FUENTES ${ }^{1}$, Víctor Manuel LÓPEZ-PASTOR ${ }^{2}$, \\ Bastian CARTER THUILLIER ${ }^{3}$.
}

\author{
${ }^{1}$ Universidad de Los Lagos, Osorno (Chile). ${ }^{2}$ Universidad de Valladolid (España). ${ }^{3}$ \\ Universidad Católica de Temuco, Temuco (Chile).
}

(Recibido 7 Febrero, 2017; Aceptado 18 Abril, 2017)

RESUMEN: Este estudio se centra en conocer las prácticas de evaluación formativa empleadas en el proceso de formación inicial del profesorado de Educación Física en el contexto de una Universidad Pública de Chile. Se compara la percepción de alumnado, profesorado y egresados. El estudio se llevó a cabo con una muestra de 23 alumnos de último curso, 50 egresados y 17 profesores de la carrera de pedagogía, mención en educación física. Para la obtención de datos se utilizó un cuestionario previamente validado, denominado: "Cuestionario para el estudio del sistema de evaluación en la formación inicial del profesorado de educación física", que cuenta con 63 ítems, medidos a través de una escala tipo Likert. Los resultados muestran que: (a) existe una alta coherencia interna entre los programas de asignatura y los sistemas de evaluación realmente utilizados; (b) las técnicas e instrumentos de evaluación más y menos utilizados, parecen indicar que se están llevando a cabo sistemas de evaluación formativa; (c) la escasa participación del alumnado en la evaluación. Parece necesario seguir investigando esta temática en más universidades chilenas.

Palabras claves: Evaluación Formativa, Evaluación Compartida, Formación Inicial Del Profesorado, Educación Superior, Chile.

There are formative and shared assessment in Pre-service Teacher Education in Chile? A university students, teacher and graduate perception

ABSTRACT: The paper is focused on knowing formative assessment practices used in Pre-service Physical Education Teacher Educación (PETE) in a public university of Chile. It compares the perception of students, teachers and graduates. The investigation was made with a sample of 23 students, 50 graduates and 17 physical education teacher's. To obtain the data a previously validated questionnaire was applied, named: "Questionnaire for the study of assessment systems used in preservice PETE”, which contains 63 items, evaluated by a Likert scale. 
The results are: (a) high internal coherence between programs and assessment systems; (B) the techniques and instruments more and less used in assessment, which suggests that they are carrying out formative assessment systems; (C) student has a scarce involvement in the Assessment. It seems necessary to further investigate this issue in more Chilean universities.

Keywords: Formative Assessment, Shared Assessment, Pre-Service Teacher Education, Higher Education, Chile.

Correspondencia: Francisco Javier Gallardo Fuentes, M.Sc., Departamento Ciencias de la Actividad Física, Universidad de Los Lagos, Osorno (Chile), Av. Fuchslocher 1305, Código Postal 5290000, teléfono: +5664333231, email: fgallardo@ulagos.cl.

\section{INTRODUCCIÓN}

Las experiencias innovadoras han demostrado que implicar al alumnado en los procesos evaluativos genera una mejora en sus propios procesos de aprendizaje (Brown, 2015). Teniendo esto en cuenta seria relevante plantearnos las siguientes preguntas: ¿Qué evaluamos en "Formación inicial del profesorado de educación física" (FIPEF)?; ¿Para qué y cómo evaluamos en FIPEF? Gibbs (2003, p.61) afirma que la evaluación es el arma más poderosa que tienen los profesores para influir en el modo en el que los estudiantes responden a las asignaturas y orientan el aprendizaje.

En este sentido, la evaluación formativa y compartida constituye una alternativa. La evaluación formativa es conocida como... todo proceso de constatación, valoración y toma de decisiones cuya finalidad es optimizar el proceso de enseñanza-aprendizaje que tiene lugar, desde una perspectiva humanizadora y no como mero fin calificador (López-Pastor, 2009, p. 35) y debe tener siempre presente para que ocurra un elemento básico, "el feedback" (Barrientos \& López-Pastor, 2015). Por su parte, López-Pastor (2009) nos dice que la evaluación formativa debe considerar la participación del alumnado en los procesos de evaluación, por lo que acuña el concepto denominado "evaluación compartida", en donde... la evaluación debe ser más un diálogo y una toma de decisiones mutuas y/o colectivas con el alumnado, y no tanto un proceso individual e impuesto (López-Pastor, Martínez \& Julián, 2007 , p. 10). Este tipo de evaluaciones ha mostrado resultados positivos en educación superior desde hace tiempo:

El sistema de evaluación formativa... ha sido puesto en práctica, investigado, evaluado y mejorado durante los últimos dieciséis años, demostrando su viabilidad y adecuación. Desde hace nueve años la Red Interuniversitaria de Evaluación Formativa y Compartida en Docencia Universitaria está generando una mayor difusión, experimentación, innovación e investigación sobre la temática (López-Pastor, 2012, p.124).

Existen estudios que demuestran que la evaluación formativa y compartida es idónea y factible en FIPEF (Castejón, López-Pastor, Julián \& Zaragoza, 2011; Gallardo-Fuentes \& Carter-Thuillier, 2016; López-Pastor, Pérez, Barba \& Lorente, 2016; Romero, Asún \& Chivite, 2016), ya que presenta una estrecha relación con la adquisición de competencias profesionales especificas, mediante la implicación del alumnado en los procesos evaluativos. (Delgado \& Oliver, 2009)

A pesar de los buenos resultados obtenidos en la utilización de sistemas de evaluación formativa, algunos estudios muestran que en evaluación se mantienen costumbres tradicionales 
a la hora de diseñar los instrumentos de evaluación, donde subyacen preguntas centradas en el recuerdo, como: ¿qué es?, ¿cuáles son?, ¿cómo se define?, ¿cuántos son?... (Ayala, Messing, Labbe \& Obando, 2010, p.62); o que se miden aspectos muy parciales de la competencia del alumnado y tienen una validez muy relativa a la hora de emitir juicios globales sobre el aprendizaje logrado (Ayala et al., 2010 p. 54). Por ello, hay estudios que muestran una clara diferencia de percepción entre lo que los profesores consideran que hacen en la evaluación (formativa) y lo que consideran alumnado y egresados (enfoque centrado en pruebas y/o exámenes finales) (Gutiérrez, Pérez \& Pérez, 2013; Martínez-Mínguez \& Flores, 2014; Martínez, Castejón \& Santos-Pastor, 2012). Revisando estudios acumulados sobre esta temática (Arribas, Manrique \& Tabernero, 2015; Gutiérrez et al., 2013; Hamodi, López \& López-Pastor, 2015; Romero-Martín, Castejón-Oliva \& López-Pastor, 2015; Silva \& López-Pastor, 2015; Tabernero \& Daniel, 2012), muestran divergencias entre las percepciones de alumnado, profesorado y egresados respecto a los instrumentos de evaluación que se utilizan en FIP. Estas diferencias suelen centrarse en las técnicas e instrumentos utilizados, y especialmente, entre el alumnado y egresados vs. el profesorado.

No hemos encontrado estudios específicos sobre esta temática llevados a cabo en Chile, solo estudios que buscaban vislumbrar la intencionalidad de la evaluación que se utilizaba en el nivel universitario, así como los resultados de una innovación que incorporaba la intencionalidad formativa en el proceso evaluativo (Ayala et al., 2010; Gallardo-Fuentes \& Carter-Thuillier, 2016). Pero no encontramos estudios que muestren una práctica sistemática en torno a esta temática, por lo que parece necesario explorar como es la situación en este país. Por ello, el objetivo de este estudio es saber si se están llevando cabo sistemas de evaluación formativa y compartida en la FIPEF en una universidad chilena, comparando la percepción de alumnado, profesorado y egresados.

\section{METODOLOGÍA}

La muestra estaba formada por 23 alumnos de último curso, 50 egresados de las ultimas 4 promociones y 17 profesores de la carrera de pedagogía, mención en educación física, de una universidad chilena. En la tabla 1 presentamos la distribución de la muestra en función del sexo y edad.

Tabla 1. Distribución de la Muestra en Función del Sexo (en Porcentaje) y Edad (en Años)

\begin{tabular}{cccc}
\hline & hombres & mujeres & edad \\
\hline Alumnado & $73.9 \%$ & $26,1 \%$ & $\mathrm{M}=23.4 \mathrm{y} d t=1.7$ \\
\hline Egresados & $60 \%$ & $40 \%$ & $\mathrm{M}=27.7 \mathrm{y} d t=3.1$ \\
\hline Profesorado & $65 \%$ & $35 \%$ & $\mathrm{M}=39.5 \mathrm{y} d t=7.8$ \\
\hline
\end{tabular}

Para la selección de la muestra de alumnado y egresados se utilizó un muestreo no probabilístico intencional, al conocer previamente las características de los individuos de la muestra (Alaminos \& Castejón, 2006). El cuestionario dirigido al alumnado de FIPEF se aplico en la última semana de clase de una de las asignaturas presentes en el semestre correspondiente. 
A los egresados se les aplico el instrumento a modo de muestreo aleatorio entre todos los egresados de los últimos 4 años, visitándolos en sus trabajos y en la reunión anual de egresados que celebra la universidad en el mes de agosto.

El profesorado participante responde a la totalidad de la dotación docente que impartía docencia en los distintos niveles de la carrera al momento de la realización del estudio, independiente del grado académico que poseían, experiencia universitaria o vinculación contractual con la Universidad.

El instrumento de medida utilizado correspondió a un cuestionario previamente validado en concordancia con los criterios de Thurstone y Likert, con un análisis de fiabilidad de $\alpha$ de Cronbach de .866 en alumnos y .821 en profesores (Ruiz-Gallardo, Ruiz \& Ureña, 2013), denominado "Cuestionario para el estudio del sistema de evaluación en la formación inicial del profesorado de educación física". El cuestionario está compuesto por un total de 63 ítems referentes a diferentes aspectos de los sistemas de evaluación y calificación utilizados a lo largo de la carrera en una escala tipo likert de cinco puntos y donde $0=$ ninguna o ninguno; $1=$ pocas o poco; $2=$ algunas o medio; $3=$ bastantes o alto y $4=$ todas o muy alto.

El cuestionario se presento en tres versiones dirigidas a cada una de las muestras (alumnado; egresados y profesorado), contando con las mismas preguntas.

\section{Análisis de Datos}

Para el análisis de datos se utilizó estadística descriptiva por variable, obteniendo la desviación típica y media aritmética de los grados de acuerdo a las afirmaciones contenidas en el instrumento por parte del alumnado, egresados y profesorado. También se llevó a cabo un análisis estadístico inferencial, concretamente diferencia de medias, a través de la prueba $U$ de Mann-Whitney, para cada combinación posible entre los tres grupos, con un nivel de significación de $p \leq .05$. Los análisis de datos se realizaron con el programa SPSS.18.0.

\section{RESULTADOS}

En las tablas 2 y 3 se presentan los datos relativos al sistema de evaluación utilizado y su coherencia con los contenidos de la asignatura.

Tabla 2. Medias y dt de los Ítems Correspondientes al Sistema de Evaluación

\begin{tabular}{|c|c|c|c|c|c|c|}
\hline & \multicolumn{2}{|c|}{ Alumnado } & \multicolumn{2}{|c|}{ Egresados } & \multicolumn{2}{|c|}{ Profesorado } \\
\hline & $\mathrm{M}$ & $d t$ & $\mathrm{M}$ & $d t$ & $\mathrm{M}$ & $d t$ \\
\hline $\begin{array}{l}\text { En las asignaturas que has cursado/impartes, ¿los sistemas de } \\
\text { evaluación aplicados se han correspondido con lo recogido } \\
\text { en el programa? }\end{array}$ & 3.61 & .66 & 2.78 & .74 & 3.00 & .71 \\
\hline $\begin{array}{l}\text { A. ¿Con que frecuencia los profesores, a través del sistema } \\
\text { de evaluación empleado en las diferentes asignaturas, te han } \\
\text { informado sobre tus aprendizajes? P. ¿Con que frecuencia } \\
\text { informas al alumnado sobre sus aprendizajes, a través del } \\
\text { sistema de evaluación empleado? }\end{array}$ & 3.43 & .73 & 2.26 & .80 & 2.59 & .62 \\
\hline
\end{tabular}


Como podemos ver en la tablas 2 y 3 los valores encontrados son medios y altos, lo que muestra que hay entre "algunas" y "bastantes" asignaturas en la carrera que tienen una buena coherencia interna y que utilizan sistemas de evaluación que podrían considerarse formativos. En ambos casos la valoración más alta corresponde al alumnado, con notable diferencia con las otras dos poblaciones, hasta el punto de que se observan diferencias significativas en los dos ítems, tanto entre alumnos y egresados como entre alumnos y profesores (ver tabla 3 ).

Tabla 3. Diferencia de Medias a los Ítems Correspondientes al Sistema de Evaluación

\begin{tabular}{|c|c|c|c|c|}
\hline & U A-E & Sig. & U A-P & Sig. \\
\hline $\begin{array}{l}\text { En las asignaturas que has cursado/impartes, ¿los sistemas de evaluación } \\
\text { aplicados se han correspondido con lo recogido en el programa? }\end{array}$ & 246.5 & $.000 *$ & 109.0 & $.010 *$ \\
\hline $\begin{array}{l}\text { A. ¿Con que frecuencia los profesores, a través del sistema de } \\
\text { evaluación empleado en las diferentes asignaturas, te han informado } \\
\text { sobre tus aprendizajes? P. ¿Con que frecuencia informas al alumnado } \\
\text { sobre sus aprendizajes, a través del sistema de evaluación empleado? }\end{array}$ & 181.5 & $.000 *$ & 80.5 & $.000 *$ \\
\hline umnos; E= Egresados. & .05 & & & \\
\hline
\end{tabular}

En la tabla 4 presentamos los resultados de un bloque de ítems que hacen referencia a la valoración del nivel de coherencia entre el sistema de evaluación y diferentes elementos didácticos de los programas de las asignaturas.

Tabla 4. Media y dt en los Ítems que hacen Referencia a la Coherencia entre Elementos de los Programas de las Asignaturas y el Sistema de Evaluación

\begin{tabular}{|c|c|c|c|c|c|c|}
\hline & \multicolumn{2}{|c|}{ Alumnos } & \multicolumn{2}{|c|}{ Egresados } & \multicolumn{2}{|c|}{ Profesores } \\
\hline & $\mathrm{M}$ & $d t$ & $\mathrm{M}$ & $d t$ & $\mathrm{M}$ & $d t$ \\
\hline $\begin{array}{l}\text { Entre las finalidades del programa y el sistema de } \\
\text { evaluación }\end{array}$ & 2.70 & 0.63 & 2.90 & .51 & 2.94 & .75 \\
\hline Entre los contenidos y el sistema de evaluación & 3.09 & 0.67 & 2.94 & .62 & 3.00 & .71 \\
\hline Entre las competencias docentes el sistema de evaluación & 2.57 & 0.84 & 2.76 & .82 & 2.76 & .97 \\
\hline $\begin{array}{l}\text { Entre la metodología de enseñanza y el sistema de } \\
\text { evaluación }\end{array}$ & 2.78 & 0.85 & 3.02 & .65 & 2.94 & .56 \\
\hline Entre la evaluación y la calificación obtenida & 2.87 & 0.76 & 2.92 & .75 & 2.71 & .92 \\
\hline
\end{tabular}

Los datos de la tabla 4 muestra que las medias de los cinco ítems son elevadas. Las valoraciones de las tres poblaciones son similares y sin un patrón claro, al punto de no encontrarse ninguna diferencia significativa entre ellas en ninguno de los cinco ítems.

En la tabla 5 presentamos un bloque de ítems que hacen referencia a la frecuencia de utilización de diversos procedimientos e instrumentos de evaluación en las asignaturas. 
Tabla 5. Medias y dt en la en la Frecuencia de Utilización de Diversos Procedimientos e Instrumentos de Evaluación en las Asignaturas

\begin{tabular}{lcccccc}
\hline & \multicolumn{2}{c}{ Alumnos } & \multicolumn{2}{c}{ Egresados } & \multicolumn{2}{c}{ Profesores } \\
\cline { 2 - 7 } & $\mathrm{M}$ & $d t$ & $\mathrm{M}$ & $d t$ & $\mathrm{M}$ & $d t$ \\
\hline Observación del profesor en clase (fichas de observación) & 2.35 & .78 & 2.24 & 1.06 & 1.59 & .87 \\
\hline Control de la participación en el aula (en grupos y debate) & 2.78 & .80 & 2.74 & .69 & 2.47 & 1.12 \\
& 2.61 & .99 & 3.02 & .82 & 2.65 & 1.06 \\
\hline Examen tipo test & 2.04 & 1.30 & 2.72 & 1.05 & 2.76 & .83 \\
\hline Examen de preguntas abiertas & 1.96 & 1.07 & 2.78 & 0.74 & 2.00 & 1.06 \\
\hline Examen de preguntas cortas (explicaciones breves) & 2.91 & .90 & 2.82 & .94 & 1.71 & 1.05 \\
\hline Examen de preguntas cerradas (definiciones) & 2.26 & 1.45 & 2.40 & 1.12 & 1.71 & 1.21 \\
\hline Exámenes escritos con documentos & 1.83 & 1.07 & 2.12 & 1.08 & 1.47 & 1.42 \\
\hline Exámenes orales & 2.96 & .98 & 3.40 & 0.88 & 2.65 & 1.37 \\
\hline $\begin{array}{l}\text { Pruebas prácticas carácter físico (ejercicios físicos, } \\
\text { situaciones de juego,...) }\end{array}$ & & & & & & \\
\hline Portafolios & 2.65 & .88 & 2.40 & 1.01 & 1.59 & 1.50 \\
\hline Cuadernos de campo & 1.70 & 1.11 & 2.18 & 1.14 & 1.53 & 1.50 \\
\hline Informes o trabajos escritos & 3.39 & .66 & 3.30 & .65 & 2.94 & .83 \\
\hline $\begin{array}{l}\text { Ensayos a partir de textos escritos o materiales } \\
\text { audiovisuales }\end{array}$ & 2.65 & .98 & 3.20 & 2.73 & 2.35 & 1.11 \\
\hline
\end{tabular}

Respecto a la utilización de diversos procedimientos e instrumentos de evaluación en las asignaturas, en la tabla 5 podemos ver que los más habituales parecen ser los trabajos escritos, las pruebas físicas, los exámenes escritos de preguntas abiertas y tipo test, el control de la participación en el aula y los ensayos. Los menos utilizados parecen ser los exámenes orales, los cuadernos de campo, los portafolios y la observación del profesor. Las medias son más altas en alumnos o en egresados, aunque sin un patrón claro.

Respecto a las diferencias significativas entre las poblaciones, aparecen en 11 casos (ver tabla 6). Entre alumnado y los egresados se encontraron diferencias de media significativas en 3 ítems con valoraciones más altas para los egresados: en "Examen de preguntas abiertas" (2.04 vs. 2.72), "Examen de preguntas cortas" (1.96 vs. 2.82) y "Pruebas prácticas carácter físico" (2.96 vs. 3.40). Entre el alumnado y el profesorado las diferencias significativas resultaron en 3 ítems, en los tres casos la valoración del alumnado fue considerablemente más alta: "Observación del profesor en clase" (2.35 vs. 1.59), en el "Examen de preguntas cerradas" (2.91 vs. 1.71) y en "Portafolios" (2.65 vs. 1.59). Por último, entre egresados y profesorado se dan diferencias significativas en 5 ítems, siempre con valores más altos en los egresados: "Observación del profesor en clase" (2.24 vs. 1.59), "Examen de preguntas cortas" (2.78 vs. 2.00), "Examen de preguntas cerradas" (2.82 vs. 1.71), "Pruebas prácticas de carácter físico" (3.40 vs. 2.65) y "Portafolios" (2.40 vs. 1.59). 
Tabla 6. Diferencia de Medias en la Frecuencia de Utilización de Diversos Procedimientos/Instrumentos de Evaluación en las Asignaturas por parte del Profesor

\begin{tabular}{|c|c|c|c|c|c|c|}
\hline & U A-E & Sig. & U A-P & Sig. & U E-P & Sig. \\
\hline $\begin{array}{l}\text { Observación del profesor en clase (fichas de } \\
\text { observación) }\end{array}$ & & & 106.5 & $.009 *$ & 287.5 & $.039 *$ \\
\hline Examen de preguntas abiertas & 410.5 & $.041 *$ & & & & \\
\hline Examen de preguntas cortas (explicaciones breves) & 329.0 & $.002 *$ & & & 248.0 & $.007 *$ \\
\hline Examen de preguntas cerradas (definiciones) & & & 79.5 & $.001 *$ & 194.0 & $.001 *$ \\
\hline $\begin{array}{l}\text { Pruebas prácticas carácter físico (ejercicios físicos, } \\
\text { situaciones de juego,...) }\end{array}$ & 412.5 & $.034 *$ & & & 285.0 & $.027 *$ \\
\hline Portafolios & & & 115.5 & $.024 *$ & 288.5 & $.042 *$ \\
\hline
\end{tabular}

En la tabla 7 presentamos un bloque de ítems que hacen referencia a la participación del alumnado en los procesos de evaluación y calificación de las asignaturas cursadas a lo largo de la carrera.

Tabla 7. Medias y dt en el Grado de Acuerdo con las Afirmaciones respecto a la Participación del Alumnado en el Proceso de Evaluación y Calificación de las Asignaturas Cursadas

\begin{tabular}{lcccccc}
\hline & \multicolumn{2}{c}{ Alumnos } & \multicolumn{2}{c}{ Egresados } & \multicolumn{2}{c}{ Profesores } \\
\cline { 2 - 7 } & $\mathrm{M}$ & $d t$ & $\mathrm{M}$ & $d t$ & $\mathrm{M}$ & $d t$ \\
\hline La calificación la decide el profesorado a partir de la evaluación & 3.13 & .87 & 2.84 & .89 & 2.41 & 1.18 \\
\hline El alumnado se autocalifica (parcial o totalmente) & 2.87 & 1.06 & 2.46 & .95 & 2.29 & .92 \\
\hline $\begin{array}{l}\text { Se califica de forma dialogada y consensuada (entre profesorado } \\
\text { y alumnado) (parcial o totalmente) }\end{array}$ & 2.22 & 1.31 & 2.10 & .18 & 2.12 & 1.22 \\
\hline Se califica a partir de la autoevaluación (parcial o totalmente) & 2.13 & 1.14 & 2.40 & .83 & 1.88 & 1.32 \\
\hline $\begin{array}{l}\text { Se califica a partir de la coevaluación (entre compañeros/as) } \\
\text { (parcial o totalmente) }\end{array}$ & 2.13 & .92 & 2.48 & .93 & 1.82 & 1.24 \\
\hline
\end{tabular}

En la tabla 7 se ve el predominio de asignaturas donde la calificación la decide directamente el profesor, aunque, paradójicamente también obtiene un valor alto la afirmación de que el alumnado se autocalifica parcial o totalmente. Los valores más bajos se dan en el ítem que hace referencia a que se califica a partir de la coevaluación entre alumnos, aunque los valores siguen siendo altos. En todos los ítems las medias más altas son del alumnado o los egresados, a veces con diferencias notables respecto al profesorado, aunque como podemos ver en la tabla 8, sólo se dan diferencias significativas en dos casos. En uno la valoración del alumnado es considerablemente más alta que la del profesorado: "La calificación la decide el profesorado a partir de la evaluación" (3.13 vs. 2.41) y en el segundo caso la valoración de los egresados es considerablemente más alta que la del profesorado: "Se califica a partir de la coevaluación entre alumnos" (2.48 vs. 1.82). En ambos casos las diferencias significativas se dan al correlacionar egresados y alumnado con profesores. 
Tabla 8. Diferencia de Medias en el Grado de Acuerdo con las Afirmaciones respecto a la Participación del Alumnado en el Proceso de Calificación de las Asignaturas Cursadas

\begin{tabular}{lllll}
\hline & U A-P & Sig. & U E-P & Sig. \\
\hline La calificación la decide el profesorado a partir de la evaluación & 127.5 & $\mathbf{. 0 4 8}$ & & \\
\hline $\begin{array}{l}\text { Se califica a partir de la coevaluación (entre compañeros/as) } \\
\text { (parcial o totalmente) }\end{array}$ & & & 285.0 & $\mathbf{. 0 3 6}^{*}$ \\
\hline U= U de Mann-Whitney; P= Profesores; A= Alumnos; E= Egresados. * $p \leq .05$ & & & \\
\hline
\end{tabular}

\section{DISCUSIÓN}

Hemos encontrado medias altas en el grado de cumplimiento del sistema de evaluación marcado en el programa (2.78-3.61) y en la utilización de procesos de evaluación formativa en las asignaturas (2.26-3.43). En ambos casos, la valoración más alta corresponde al alumnado, con diferencias significativas en los dos ítems, tanto respecto a los egresados como al profesorado. Estos resultados son similares a los encontrados en un estudio con alumnado universitario español quienes indicaron que tanto "la correspondencia de los sistemas de evaluación aplicados y lo contenido en los programas de asignatura" como "la frecuencia de información sobre los aprendizajes a través del sistema de evaluación de las asignaturas" ocurre entre algunas y bastantes veces, aunque para el estudio español la valoración arrojo diferencias significativas siendo más alta la del profesorado (Gutiérrez, Pérez \& Pérez, 2013).

Los resultados indican una elevada coherencia entre los elementos didácticos de los programas de las asignaturas (finalidades, contenidos, competencias, metodología y calificación) y el sistema de evaluación (2.57-3.09); además de un elevado grado de acuerdo entre las tres poblaciones, dado que no hay diferencias significativas entre los tres colectivos. En cambio, en un estudio similar (Gutiérrez et al., 2013) se encontraron diferencias significativas entre las poblaciones, en donde siempre la valoración del profesorado fue más alta que el alumnado y los egresados. También aparecen diferencias en los estudios (Ayala et al., 2010; Gutiérrez-García, Pérez-Pueyo, Pérez-Gutiérrez \& Palacios-Picos, 2011; Martínez et al., 2012; Ruiz-Gallardo et al., 2013)

Respecto a la utilización de los diferentes procedimientos e instrumentos de evaluación en las asignaturas, las medias son muy variadas, desde puntuaciones bajas (1.53), hasta puntuaciones muy altas (3.40). Los ítems de este apartado arrojaron diferencias significativas entre las 3 poblaciones en 11 casos. Al igual que en otros estudios los instrumentos más utilizados suelen ser "trabajos escritos, las pruebas físicas, los exámenes escritos de preguntas abiertas y los exámenes tipo test" (Gutiérrez et al., 2013; Martínez et al., 2012), mientras que entre los menos utilizados están los exámenes orales, en concordancia con (Arribas et al., 2015; Palacios-Picos, López-Pastor \& Barba-Martín, 2013; Ruiz-Gallardo et al., 2013). Por otro lado se puede ver que en la mayoría de los ítems se observaron diferencias significativas entre las tres poblaciones, pero sin seguir ningún patrón fijo; a diferencia de estudios, en los que las diferencias significativas se dan entre el profesorado respecto a alumnado y egresados (Gutiérrez-García et al., 2011; Martínez-Mínguez \& Flores, 2014; Martínez et al., 2012).

Respecto a la participación del alumnado en el proceso de evaluación y calificación de las asignaturas cursadas, los resultados vuelven a mostrar una gran diversidad de medias 
(Desde 1.82 hasta 3.13). Aparecen diferencias significativas sólo en dos ítems. El primero hace referencia a si la calificación la decide sólo el profesor. El alumnado considera que sí en un porcentaje muy elevado, mientras que el profesorado muestra una media más baja (3.13 vs. 2.41). Por tanto, desde el punto de vista del alumnado en la mayoría de las asignaturas sigue predominando el modelo tradicional, donde la decisión de la calificación la tiene solo el profesor, como denunciaban hace casi dos décadas Trillo \& Porto (1999) y que aun se puede observar en otros estudios (Ayala et al., 2010; Silva \& López-Pastor, 2015; Tabernero \& Daniel, 2012), aunque se pueden apreciar múltiples avances en la incorporación de sistemas de evaluación formativa con resultados positivos en el alumnado (Brown, 2015; Barrientos \& López-Pastor, 2015; Castejón et al., 2011; Gallardo-Fuentes \& Carter-Thuillier, 2016).

En cambio, la valoración del profesorado podría mostrar una cierta evolución y cambio en este sentido, dado que parece que cada vez es mayor el porcentaje de profesorado que implica al alumno en los procesos de evaluación y calificación, tal como parece estar ocurriendo en la última década en la FIP en España, según los estudios de (Palacios-Picos \& López-Pastor, 2013; Romero-Martín et al., 2015; Romero-Martín et al., 2016). Sin embargo la tendencia a evaluar de forma tradicional sigue instalada, cumpliendo las tipologías de profesorado universitario según los métodos evaluativos que utilizan ("profesorado innovador", "profesorado tradicional" y "profesorado ecléctico") establecidas por Hamodi et al. (2015), Palacios \& López-Pastor (2013) y Palacios et al. (2013).

Por otro lado, la valoración de los egresados es considerablemente más alta que la del profesorado en el ítem que hace referencia a la coevaluación entre alumnos (2.48 vs. 1.82). Este resultado es extraño, dado que parece indicar que los egresados consideran que había más situaciones de este tipo cuando ellos estudiaron que las que hay en estos momentos en opinión del profesorado. Estos resultados, sin tener una diferencia significativa, se comportan igual a lo encontrado en Gutiérrez-García et al. (2011). Una posible explicación sería que los egresados no han entendido correctamente que significa el concepto coevaluación.

\section{CONCLUSIONES}

Los resultados muestran que sí se están llevando a cabo sistemas de evaluación formativa en la FIPEF en la universidad chilena en que se lleva a cabo el estudio. En lo referente a los procedimientos e instrumentos más utilizados, aparecen diferencias significativas de percepción entre los colectivos involucrados (alumnado, egresados y profesorado) y un predominio en el uso de instrumentos que no involucran al alumnado en la evaluación. Parece quedar mucho camino que recorrer en lo que respecta a la implicación del alumnado en los procesos de evaluación (autoevaluación, evaluación entre pares y evaluación compartida), en donde la percepción de los colectivos involucrados difiere significativamente entre alumnado y profesorado.

El presente artículo puede ser un aporte para el espacio educativo universitario en Chile y específicamente para la FIPEF, considerando la falta de investigación encontrada sobre la temática. Además, este estudio puede ser un punto de partida para procesos de innovación en el aula y futuras investigaciones, en un momento en que la universidad chilena está adoptando el currículum por competencias. 
En futuros estudios sería importante indagar en los efectos que la implementación de procesos de evaluación formativa y compartida podría tener en la percepción del alumnado y el profesorado chileno de FIP, así como en aspectos como rendimiento académico, satisfacción y carga de trabajo para alumnado y profesorado.

\section{AGRADECIMIENTOS}

Este estudio se ha llevado a cabo dentro del proyecto de I+D+i: "La competencias docentes en la formación inicial del profesorado de educación física". Programa Nacional de Investigación y Desarrollo del Gobierno Español 2013-2016. Referencia: EDU 2013-42024-R. Duración: 3 años (2014-2016).

También agradecemos su apoyo al departamento de Ciencias de la Actividad física y a la Escuela de Pedagogía de la Universidad de los Lagos.

\section{Referencias}

Alaminos, A. y Castejón, J. (2006). Elaboración, análisis e interpretación de encuestas, cuestionarios y escalas de opinión. Alicante: Marfil.

Arribas, J. M., Manrique, J. y Tabernero, B. (2015). Instrumentos de evaluación utilizados en la formación inicial del profesorado y su coherencia para el desarrollo de competencias profesionales en los estudiantes: visión del alumnado, egresados y profesorado. Revista Complutense de Educación, 27(1), 237-225.

Ayala, R., Messing, H., Labbé, C. y Obando, N. (2010). Congruencia entre el diseño curricular y la evaluación de los aprendizajes esperados en cátedras impartidas en una universidad chilena. Estudios pedagógicos, 36(1), 53-67.

Barrientos, E. y López-Pastor, V. (2015). La evaluación formativa en educación superior. Una revisión internacional. Revista arbitrada del CIEG. 21, 272-284.

Brown, S. (2015). La evaluación auténtica: el uso de la evaluación para ayudar a los estudiantes a aprender. RELIEVE-Revista Electrónica de Investigación y Evaluación Educativa, 21(2).

Castejón, F., López-Pastor, V., Julián, J. y Zaragoza, J. (2011). Evaluación formativa y rendimiento académico en la formación inicial del profesorado de educación física. International Journal of Medicine and Science of Physical Activity and Sport, 11(42), 238-346.

Delgado, A. y Oliver, R. (2009). Interacción entre la evaluación continua y la autoevaluación formativa: La potenciación del aprendizaje autónomo. Revista de docencia universitaria, (4).

Hamodi, C., López, A. y López-Pastor, V. (2015). Red de evaluación formativa y compartida en docencia universitaria: creación, consolidación y líneas de trabajo. REVALUE, 3(1).

Gallardo-Fuentes, F. y Carter-Thuillier, B. La evaluación formativa y compartida durante el prácticum en la formación inicial del profesorado: Análisis de un caso en Chile. Retos. Nuevas tendencias en Educación Física Deportes y Recreación, (29), 258-263. 
Gibbs, G. (2003). Uso estratégico de la educación en el aprendizaje: En Brown, S. y Glasner, A. (Coord.). Evaluar en la universidad: problemas y nuevos enfoques (pp. 61-74). Madrid: Narcea.

Gutiérrez, C., Pérez, P. y Pérez, M. (2013). Percepciones de profesores, alumnos y egresados sobre los sistemas de evaluación en estudios universitarios de formación del profesorado de educación física. Ágora para la Educación Física y el Deporte, 15(2), 130-151.

Gutiérrez-García, C., Pérez-Pueyo, Á., Pérez-Gutiérrez, M. y Palacios-Picos, A. (2011). Percepciones de profesores y alumnos sobre la enseñanza, evaluación y desarrollo de competencias en estudios universitarios de formación de profesorado. Cultura y Educación, 23(4), 499-514.

López-Pastor, V. (Coord.) (2009). La Evaluación Formativa y Compartida en Educación Superior: propuestas, técnicas, instrumentos y experiencias. Madrid: Narcea.

López-Pastor, V., Martínez, L. y Julián, J. (2007). La Red de Evaluación Formativa, Docencia Universitaria y Espacio Europeo de Educación Superior (EEES). Presentación del proyecto, grado de desarrollo y primeros resultados. REDU. Revista de Docencia Universitaria, 5(2), 1-19.

López-Pastor, V., Pérez, A., Barba, J. y Lorente, E. (2016). Percepción del alumnado sobre la utilización de una escala graduada para la autoevaluación y coevaluación de trabajos escritos en la formación inicial del profesorado de educación física (FIPEF). CCD. Cultura Ciencia Deporte, (31), 37-50.

López-Pastor, V. (2012). Evaluación formativa y compartida en la universidad: clarificación de conceptos y propuestas de intervención desde la Red Interuniversitaria de Evaluación Formativa. Psychology, Society \& Education, 4(1), 117-130.

Martínez, L., Castejón, F. y Santos-Pastor, M. (2012). Diferentes percepciones sobre evaluación formativa entre profesorado y alumnado en formación inicial en educación física. Revista electrónica interuniversitaria de formación del profesorado, 15(4), 57-67.

Martínez-Mínguez, L. y Flores, G. (2014). Profesorado y egresados ante los sistemas de evaluación del alumnado en la formación inicial del maestro de educación infantil. RIDU, 8(1), 2.

Palacios-Picos, A. y López-Pastor, V. (2013). Haz lo que yo digo pero no lo que yo hago: sistemas de evaluación del alumnado en la formación inicial del profesorado. Revista de educación, (361), 279-305.

Palacios-Picos, A., López-Pastor, V. y Barba-Martín, J. (2013). Tipologías de profesorado universitario en función de la evaluación aplicada a los futuros docentes. Revista Estudios sobre Educación, (23). 173-195.

Romero, M., Asún, S. y Chivite, S. (2016). La autoevaluación en expresión corporal en formación inicial del profesorado de educación física: un ejemplo de buena práctica. Retos. Nuevas tendencias en Educación Física Deportes y Recreación, (29), 236-241.

Romero-Martín, M., Castejón-Oliva, F. y López-Pastor, V. (2015). Divergencias del alumnado y del profesorado universitario sobre las dificultades para aplicar la evaluación formativa. RELIEVE-Revista Electrónica de Investigación y Evaluación Educativa, 21(1). 
Ruiz-Gallardo, J., Ruiz, E. y Ureña, N. (2013). La evaluación en la formación inicial del profesorado: qué creemos hacer y qué perciben los alumnos. CCD. Cultura Ciencia Deporte, 8(22), 17-29.

Silva, I. y López-Pastor, V. (2015). ¿Cómo vive el alumnado la evaluación en formación inicial del profesorado? @tic. revista d'innovació educativa, (14), 90-100.

Tabernero, B. y Daniel, M. (2012). Análisis de los sistemas de evaluación del alumnado en la formación del profesorado: estudio comparativo entre lo que opinan profesores y egresados. REIFOP. Revista electrónica interuniversitaria de formación del profesorado, 15 (3)

Trillo, F y Porto, M. (1999). La percepción de los estudiantes sobre su evaluación en la universidad. Revista Innovación Educativa, (9) 55-75. 